\title{
Cost Effectiveness of a Cultural Physical Activity Intervention to Reduce Blood Pressure Among Native Hawaiians with Hypertension
}

\author{
Ashley F. Railey ${ }^{1,2}$ - Clemma Muller ${ }^{1} \cdot$ Carolyn Noonan $^{1} \cdot$ Maureen Schmitter-Edgecombe $^{3} \cdot$ Ka'imi Sinclair $^{1}$. \\ Corin $\mathrm{Kim}^{4} \cdot$ Mele Look $^{5} \cdot$ J. Keawe'aimoku Kaholokula ${ }^{5}$
}

Accepted: 19 July 2021 / Published online: 13 August 2021

(c) The Author(s) 2021

\begin{abstract}
Objective The aim of this study was to calculate the costs and assess whether a culturally grounded physical activity intervention offered through community-based organizations is cost effective in reducing blood pressure among Native Hawaiian adults with hypertension.

Methods Six community-based organizations in Hawai 'i completed a randomized controlled trial between 2015 and 2019. Overall, 263 Native Hawaiian adults with uncontrolled hypertension $(\geq 140 \mathrm{mmHg}$ systolic, $\geq 90 \mathrm{mmHg}$ diastolic) were randomized to either a 12-month intervention group of hula (traditional Hawaiian dance) lessons and self-regulation classes, or to an education-only waitlist control group. The primary outcome was change in systolic blood pressure collected at baseline and 3, 6, and 12 months for the intervention compared with the control group. Incremental cost-effectiveness ratios (ICERs) were calculated for primary and secondary outcomes. Non-parametric bootstrapping and sensitivity analyses evaluated uncertainty in parameters and outcomes.

Results The mean intervention cost was US $\$ 361 /$ person, and the 6-month ICER was US $\$ 103 / \mathrm{mmHg}$ reduction in systolic blood pressure and US\$95/mmHg in diastolic blood pressure. At 12 months, the intervention group maintained reductions in blood pressure, which exceeded reductions for usual care based on blood pressure outcomes. The change in blood pressure at 12 months resulted in ICERs of US $\$ 100 / \mathrm{mmHg}$ reduction in systolic blood pressure and US $\$ 93 / \mathrm{mmHg}$ in diastolic blood pressure. Sensitivity analyses suggested that at the estimated intervention cost, the probability that the program would lower systolic blood pressure by $5 \mathrm{mmHg}$ was 67 and $2.5 \%$ at 6 and 12 months, respectively.

Conclusion The 6-month Ola Hou program may be cost effective for low-resource community-based organizations. Maintenance of blood pressure reductions at 6 and 12 months in the intervention group contributed to potential cost effectiveness. Future studies should further evaluate the cost effectiveness of indigenous physical activity programs in similar settings and by modeling lifetime costs and quality-adjusted life-years.
\end{abstract}

Trial Registration Number NCT02620709.

Ashley F. Railey

arailey@iu.edu

1 Institute for Research and Education to Advance Community Health (IREACH), Elson S. Floyd College of Medicine, Washington State University, Spokane, WA, USA

2 Department of Sociology, Indiana University, 1022 E. Third St, Bloomington, IN 47405-7103, USA

3 Department of Psychology, Washington State University, Pullman, WA, USA

4 Kilohana, University of Hawai 'i at Hilo, Hilo, HI, USA

5 Department of Native Health, John A. Burns School of Medicine, University of Hawai 'i at Mānoa, Mānoa, HI, USA

\section{Introduction}

High blood pressure is a primary risk factor for cardiovascular disease and cerebrovascular diseases, such as coronary heart disease and stroke. The prevalence of hypertension in Native Hawaiians is nearly $48 \%$, compared with $23 \%$ in White Americans, 38\% in Japanese Americans, and 30\% in Filipino Americans [1, 2]. Native Hawaiians are also 30\% less likely to have controlled hypertension [3]. Native Hawaiian people experience cardiovascular disease and stroke an average of 10 years earlier [4], with mortality rates that are 68 and 20\% higher than in White people, respectively [5]. Despite the increased risk, Native Hawaiian people are less 


\section{Key Points for Decision Makers}

This study used outcomes from a randomized controlled trial that showed the efficacy of cultural physical activity in reducing blood pressure among Native Hawaiian adults with hypertension to define the cost implications for resource-limited community-based organizations in Hawai 'i.

Assessing the costs and cost effectiveness helps establish the role of cultural physical activity interventions in addressing community-specific hypertension burden.

Results are limited by the 12-month timeline and narrow cost perspective, but show evidence that indigenous forms of physical activity may be cost effective in reducing blood pressure in Native Hawaiian adults.

Further research is needed to explore the long-term economic impact of the intervention, including patient costs and potential medication changes.

likely to receive adequate treatment for cardiovascular conditions than Whites people [1-6].

The American Heart Association recommends combining pharmacological therapy with lifestyle changes, including physical activity, to reduce high blood pressure [7]. Native Hawaiians in Hawai' 'i have reported that they prefer to engage in culturally grounded physical activities that are group-based and incorporate traditional practices instead of Western activities, such as biking or running [8]. To improve blood pressure control in Native Hawaiian adults with previously uncontrolled hypertension, the KāHOLO Project recently conducted a trial to determine the efficacy of a 6-month intervention (called Ola Hou i ka Hula or Ola Hou) that included hula practice and self-regulation training. Hula is a traditional dance and hallmark of Native Hawaiian culture that can also meet the recommendations for moderateand high-intensity physical activity $[9,10]$. The KāHOLO Project was funded by the National Heart, Lung and Blood Institute from 2015 to 2020 [11]. During the 6-month intervention, retention rates approached $80 \%$ and intervention participants experienced significant improvements in blood pressure and reduced their cardiovascular disease risk [9]. These results have stimulated interest in continuing the intervention in Native Hawaiian communities.

Translating an intervention into a community program as standard care often requires defining the intervention costs, along with outcomes. Cost-effectiveness analyses evaluate the effectiveness of the program or intervention relative to the cost of implementation, to provide policymakers and healthcare administrators with a common metric for comparing across types of interventions and programs [12]. Treating hypertension is generally found to be cost effective or cost saving [13-15]. This includes health education and physical activity interventions that rely on community health workers to shift tasks away from physicians and pharmacists [16-18]. While the Ola Hou intervention employed similar cost reduction measures, the intervention would likely be implemented at federally funded community health centers, which are the safety nets of the US health care system and operate under limited budgets [19]. To continue serving their populations who rely on these centers for the majority of their health needs, every community health center program requires justification of their programs by weighing the benefits against the costs.

The objective of this study was to calculate the costs and estimate the cost effectiveness associated with the reduction in blood pressure from participation in the culturally grounded Ola Hou intervention. We evaluated the costs from the provider perspective of the community health centers and other health-focused organizations in Hawai ' $i$ that would provide the program. Taking this perspective influenced the timeline and intention-to-treat approach of the analysis. The timeline included the 6-month intervention analysis to posit the health benefits of Ola Hou i ka Hula in comparative measures with other interventions that reduce blood pressure. We then extended the analysis to predict the persistence of Ola Hou i ka Hula on controlling blood pressure at 12 months.

\section{Methods}

\subsection{Ola Hou i ka Hula (Restoring Health Through Hula) Intervention}

Hula is the traditional dance of Hawai ' $i$ and its indigenous people that employs choreographed, synchronized, rhythmic body movements to illustrate the stories and meanings of the accompanying songs and chants [20,21]. The 6-month Ola Hou i ka Hula (Ola Hou henceforth) intervention was a randomized controlled trial (RCT) that assessed hula training and self-regulation education on blood pressure reduction in Native Hawaiian adults with uncontrolled blood pressure ( $\geq 140 \mathrm{mmHg}$ systolic, $\geq 90 \mathrm{mmHg}$ diastolic) but no cardiovascular disease. Six community-based organizations in Hawai' $i$ implemented the trial, of which one was a federally qualified health center, three were nonprofit community centers, and two were part of the Native Hawaiian Health Care System [22]. The community-based organizations were involved in refining the outcome and cost parameters, as well as interpretation within the context of the communities. The 
trial design, participants, intervention design, outcomes, and ethics approvals have been described in detail elsewhere (in print) [11,23].

Briefly, 263 participants received the intervention between 2015 and 2019 (131 Ola Hou participants and 132 control participants). All participants received a brief heart health education that included three $1 \mathrm{~h}$ lessons over 1 week. Following this education, participants were placed in their randomly assigned groups. The first 3-months (12 weeks) of the intervention group consisted of hula training, with two 60-min classes per week led by a Kumu Hula (trained instructor). In months 3-6, weekly sessions with a community peer educator provided culturally relevant hypertension management education, guidance on individual goal setting, and taught self-regulation strategies. These sessions in months 3-6 were accompanied by self-directed hula practice sessions and three hula training classes led by a Kumu Hula. The education-only control group (controls) were offered hula training at months 6 through 12 . Class sizes ranged from 10 to 15 participants across sites and intervention arms.

Participants completed clinical measures and questionnaires at baseline and 3, 6, and 12 months that included blood pressure, medication records, cardiovascular disease risk factors, and psychological and behavioral risk factors. No participants in the control group completed the clinical measures and questionnaires at the 12-month timeline because they were offered the hula training at months 6 through 12.

\subsection{Effectiveness}

The primary outcome of the intervention was a reduction in systolic blood pressure among Native Hawaiian adults with uncontrolled hypertension $(\geq 140 \mathrm{mmHg}$ systolic, $\geq 90 \mathrm{mmHg}$ diastolic). Reductions in diastolic blood pressure were secondary. Blood pressure was taken following standard protocols before completing the questionnaires [24]. Three separate blood pressure measures were taken at each data collection period and the last two were averaged to calculate the mean for each participant. The last observation carried forward approach addressed missing values in blood pressure readings ( $n=5$ in each group) for the 6-month within-trial observations.

To evaluate the persistence of effects beyond the hula and educational components of the intervention, we predicted the 12-month blood pressure readings for the control group. We used the difference in blood pressure from the control group at baseline to 6 months to predict the difference in blood pressure from 6 to 12 months. As predicted values, the difference between 6 and 12 months for the control group equated to a proxy for a control group with 6 months of no participation in the Ola Hou program.

\subsection{Costs}

The cost analysis was conducted from the standpoint of the community organization to approximate the cost of offering this program, and aligned with the Consolidated Health Economic Evaluation Reporting Standards (CHEERS) reporting guidelines $[12,25]$. Only costs essential to implementing the program, instead of costs involved in research protocols, such as intensive recruiting and participant compensation, were included. Implementation costs came from detailed program records of resources used, salary rates, and prices of materials. All costs were reported in 2015 dollars. Discounting was not applied given the 12-month duration of the analysis.

The Ola Hou costs covered program recruitment, educational materials, salaries, and facilities to manage and use the space. Upon implementation, recruitment would rely on physician referrals and word of mouth, along with limited flyers around the communities. Educational materials and personnel were divided by activity (hula or self-regulation). Kumu Hula rates reflected the expected costs per hour for a trained hula instructor in Hawai'i. Hourly rates for the self-regulation classes included fringe benefits for the community peer educator and covered additional time for class preparation and administrative activities. Facility costs represented the cost per square foot to use the space, at market value. For community-based organizations that own the space, this captures the opportunity cost of forgoing similar programs, and contributes to overhead costs [26]. Participant medical costs for specialized care, physician visits, and other health-related expenditures were not collected during the intervention timeline. Both the Ola Hou and the control group received three $1 \mathrm{~h}$ lessons of culturally tailored heart health education before the intervention. Additional details are provided in the primary RCT manuscript [23]. However, in practice, those not enrolled in Ola Hou would incur no programmatic costs as no other program is offered in usual care.

\subsection{Analysis Procedure}

The primary aims of our analysis were to define the costs of the Ola Hou intervention and the cost effectiveness of the intervention in reducing systolic blood pressure among Native Hawaiians with hypertension. Incremental costeffectiveness analyses were also calculated for diastolic blood pressure. We evaluated the higher-bound costs and cost effectiveness of the program by basing the estimations on a class size of 10 . The incremental cost-effectiveness ratio (ICER) was calculated as the incremental cost of the Ola Hou intervention relative to the education-only control group, divided by each measure of the incremental effectiveness of the intervention relative to the control group. 
The ICER represented the change in cost per reduction in outcome over the given time period and corresponding with the RCT activities. Baseline to 6 months was the primary time period, but we additionally calculated ICERs for baseline to 3 months (predominantly the hula component), 3-6 months (self-regulation component), and baseline to 12 months (predicted maintenance of reduced blood pressure). We adopted an intention-to-treat approach for a total of 263 participants (131 Ola Hou, 132 controls). Retention at 6 months was assessed for those with complete baseline and follow-up data.

A range of analyses examined the differential impacts of key parameters on the costs and effectiveness of the program. We captured uncertainty in the ICERs with $95 \%$ confidence intervals through non-parametric bootstrap resampling of the data [27]. We then varied the program costs by $20 \%$ in either direction to capture variable costs attributed to training/orientation for the instructors, potential recruitment strategies, and facilities. Without a standard willingness to pay per reduction in blood pressure, we evaluated the probability that the intervention would be effective in reducing blood pressure at clinically meaningful levels. We relied on clinical standards for a meaningful reduction in systolic (diastolic) blood pressure at $5 \mathrm{mmHg}$ $[28,29]$. Finally, we used the estimated costs to examine the per-person costs based on expected class sizes $(5,10$, 15 , and 20).

\section{Results}

The baseline characteristics of the participants, by study arm, are shown in Table 1. The average age was 56 years, with a baseline blood pressure reading of $144 / 85 \mathrm{mmHg}$ in the Ola Hou group and $145 / 85 \mathrm{mmHg}$ in the control group. In both groups, females made up the majority of participants (85\% Ola Hou, $83 \%$ controls) and over $70 \%$ previously danced hula (75\% Ola Hou, 71\% controls). Medical conditions associated with high blood pressure included type 2 diabetes (40\% Ola Hou, 50\% controls) and high cholesterol (49\% Ola Hou, 52\% controls). Almost $90 \%$ of participants had been prescribed antihypertensive medications before the intervention (87\% Ola Hou, $86 \%$ controls) and continued medications throughout the 6-month follow-up. At the end of 6 months, complete cases accounted for 105/131 (80\%) initial participants in the Ola Hou group and 112/132 (85\%) education-only control group participants.

The various costs of the program appear in Table 2. The total cost of the Ola Hou intervention for 6 months totaled US $\$ 3691$, which included US\$2958 for the first 3 months and US\$733 for the 3- to 6-month period.

Table 3 presents the estimated incremental programmatic costs, changes in blood pressure, and the corresponding ICERs for each time period of the intervention for a class size of 10 participants. The average programmatic cost of the Ola Hou group compared with the control group was US\$361/person for the 6-month intervention. On average, the Ola Hou group reduced systolic blood pressure by $3.5 \mathrm{mmHg}$ ( $3.8 \mathrm{mmHg}$ diastolic) more than the control group throughout the 6-month intervention. This translates
Table 1 Characteristics of participants, by study arm at baseline

\begin{tabular}{lll}
\hline Variable & Ola Hou $[n=131]$ & Controls $[n=132]$ \\
\hline Age, years [mean (SD)] & $58.1(13.7)$ & $57.9(12.6)$ \\
Female & $111(84.7)$ & $109(82.6)$ \\
Danced hula & $98(74.8)$ & $93(70.5)$ \\
Other medical conditions & & \\
High cholesterol & $64(48.9)$ & $69(52.3)$ \\
Diabetes & $52(39.7)$ & $66(50.0)$ \\
Other medical conditions & $18(13.7)$ & $25(18.9)$ \\
No other medical condition & $36(27.5)$ & $28(21.2)$ \\
Prescribed hypertension medications & $114(87.0)$ & $113(85.6)$ \\
No. of hypertension medications [mean (SD)] & $1.31(0.88)$ & $1.40(0.87)$ \\
Systolic blood pressure, mmHg [mean (SD)] & $144.1(16.9)$ & $145.1(13.9)$ \\
Diastolic blood pressure, mmHg [mean (SD)] & $85.2(12.6)$ & $84.5(10.4)$ \\
Retention at 6 months & $105(0.80)$ & $112(0.85)$ \\
\hline
\end{tabular}

Data are expressed as $n(\%)$ unless otherwise specified. Numbers for specific variables may not equate to the total sample size because of missing data. Group difference $p$ values were based on two-sample independent $t$ tests and Chi-square or Fisher's exact tests, as appropriate

$S D$ standard deviation 
Table 2 Description of costs for the 6-month Ola Hou

\begin{tabular}{|c|c|c|c|}
\hline Programmatic costs & Unit & Total cost (US\$) & Notes and source \\
\hline \multicolumn{4}{|l|}{ Recruitment } \\
\hline Print/flyers/mailers & 100 flyers & 50 & Reported by intervention \\
\hline Heart health education & $3 \mathrm{~h}$ & 81 & Reported by intervention; at US $\$ 27 / \mathrm{h}$ with fringe benefits \\
\hline \multicolumn{4}{|l|}{ Self-regulation sessions } \\
\hline Materials & US\$30/program & 450 & Reported by intervention; includes handouts and health manuals \\
\hline Educational teaching & $12 \mathrm{~h}$ & 324 & Reported by intervention; at US $\$ 27 / \mathrm{h}$ with fringe benefits \\
\hline \multicolumn{4}{|l|}{ Hula training } \\
\hline Kumu Hula teaching & $27 \mathrm{~h}$ & 2430 & Reported by intervention; at US $\$ 90 / \mathrm{h}$ \\
\hline Materials & 1 miscellaneous & 50 & Reported by intervention; includes any stereo or material fixed costs \\
\hline Training/orientation & $6 \mathrm{~h}$ & 540 & Reported by intervention; one-time training \\
\hline Facilities & $39 \mathrm{~h}$ & 78 & Estimated; cost per square foot, at market value [60] \\
\hline \multirow[t]{2}{*}{ Administration } & $4 \mathrm{~h}$ & 108 & Reported by intervention; preparation of materials at US $\$ 27 / \mathrm{h}$ \\
\hline & & US\$3691 & \\
\hline
\end{tabular}

into an ICER of US $\$ 103 / \mathrm{mmHg}$ reduction in systolic (US\$95/mmHg diastolic) blood pressure. At 12 months, the costs remain the same but we estimated a difference in systolic blood pressure of $3.6 \mathrm{mmHg}(3.9 \mathrm{mmHg}$ diastolic) for an ICER of US $\$ 100 / \mathrm{mmHg}$ reduction in systolic (US\$93/mmHg diastolic) blood pressure. Broken down by intervention component, after 3 months, the average cost was US\$288/person, the average difference in groups was $3.3 \mathrm{mmHg}$ systolic (3.4 $\mathrm{mmHg}$ diastolic), and the ICER was US\$87 per reduction in systolic blood pressure (US\$88/ $\mathrm{mmHg}$ diastolic blood pressure). From 3 to 6 months, the average cost was US $\$ 77 /$ person, the average difference in groups was $0.2 \mathrm{mmHg}$ systolic $(0.4 \mathrm{mmHg}$ diastolic), and the ICER was US $\$ 385 / \mathrm{mmHg}$ systolic blood pressure (US\$193/mmHg diastolic blood pressure).

Varying the training and implementation costs by $20 \%$ in each direction resulted in the intervention costs ranging from US\$3449 to US\$3538 for the 6-month program. Figure 1 displays the bootstrap estimates using these ranges across time periods. From this plot, the probability of the program being effective for a $5 \mathrm{mmHg}$ reduction in systolic blood pressure was 67 and $2.5 \%$ for 6 and 12 months, respectively. The diastolic results are shown in the electronic supplementary material. Figure 2 presents the results by time period and class sizes for the cost per difference in reduction of systolic blood pressure between the Ola Hou and control groups. The results for the cost per reduction in diastolic blood pressure appear in the electronic supplementary material and match the trends of those for systolic blood pressure. Decreasing per person costs resulted in a decreased cost per reduction in blood pressure. In all four time points, a statistically significant difference exists between the per person costs for a class size of 5 compared with 20 participants. For 10-20 participants, the magnitude of the per person cost shrinks with the 3 - to 6-month time period, reflecting the smallest cost per person. The smallest average difference in blood pressure between the Ola Hou and control groups also occurs during this time $(0.2 \mathrm{mmHg})$. The period that corresponds to the intervention (baseline to 6 months) was estimated to have the largest difference in blood pressure reduction, at $3.5 \mathrm{mmHg}$.
Table 3 Average costs and cost effectiveness of the 6-month intervention

\begin{tabular}{|c|c|c|c|c|c|}
\hline \multirow[t]{2}{*}{ Time period } & \multirow{2}{*}{$\begin{array}{l}\text { Programmatic cost } \\
\text { US } \$ / \text { person }\end{array}$} & \multicolumn{2}{|l|}{ Systolic } & \multicolumn{2}{|l|}{ Diastolic } \\
\hline & & $\begin{array}{l}\text { Difference, } \\
\mathrm{mmHg}\end{array}$ & $\begin{array}{l}\text { ICER, US\$/ } \\
\mathrm{mmHg}\end{array}$ & $\begin{array}{l}\text { Difference, } \\
\mathrm{mmHg}\end{array}$ & $\begin{array}{l}\text { ICER, } \\
\text { US\$/ } \\
\text { mmHg }\end{array}$ \\
\hline Baseline to 3 months & 288 & 3.3 & 87 & 3.4 & 85 \\
\hline $3-6$ months & 77 & 0.2 & 385 & 0.4 & 193 \\
\hline Baseline to 6 months & 361 & 3.5 & 103 & 3.8 & 95 \\
\hline Baseline to 12 months & 361 & 3.6 & 100 & 3.9 & 93 \\
\hline
\end{tabular}

Blood pressure measures from an intention-to-treat analysis. Cost and $\mathrm{mmHg}$ are the difference in the Ola Hou intervention group minus the control group. Blood pressure measures for the control group at 12 months are predicted values

ICER incremental cost-effectiveness ratio, $\mathrm{mmHg}$ millimeter of mercury, US\$ United States dollars 
Fig. 1 Uncertainty and sensitivity analyses for costs and incremental cost effectiveness by time period (systolic blood pressure). Reported average class size of 10 people. Bootstrap estimates using $\pm 20 \%$ range for costs. Cost per difference in the reduction of systolic blood pressure between the Ola Hou and control groups. Costs and effectiveness are incremental. $\mathrm{mmHg}$ millimeter of mercury, US\$ United States dollars, ICER incremental cost-effectiveness ratio

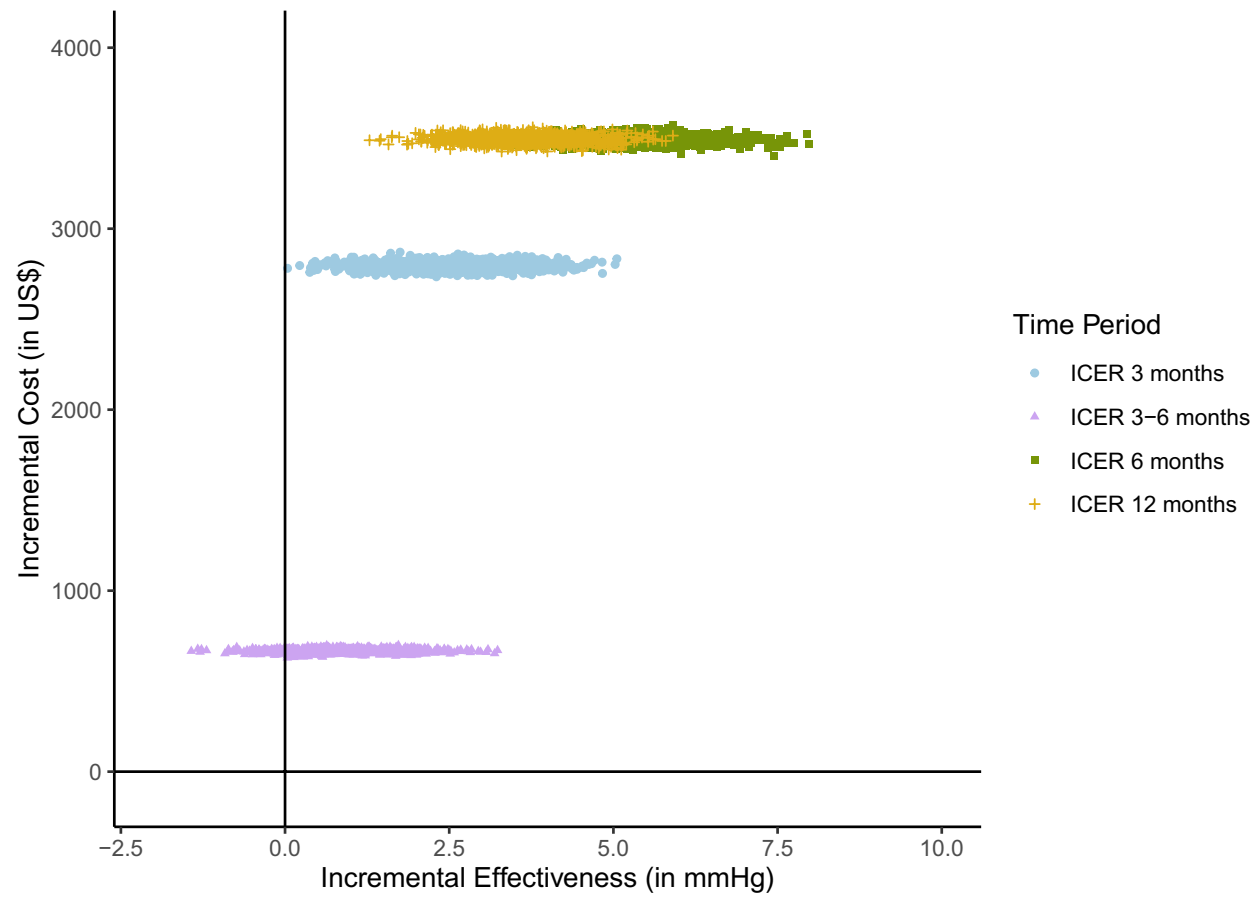

Fig. 2 Uncertainty and sensitivity analyses for costs and incremental cost effectiveness by time period and class size (systolic blood pressure). Bootstrap estimates using $\pm 20 \%$ range for costs. Cost per difference in the reduction of systolic blood pressure between the Ola Hou and control groups. Costs and effectiveness are incremental. Class sizes are based on reported expected class sizes $(5,10,15$, and 20). $\mathrm{mmHg}$ millimeter of mercury, US\$ United States dollars.
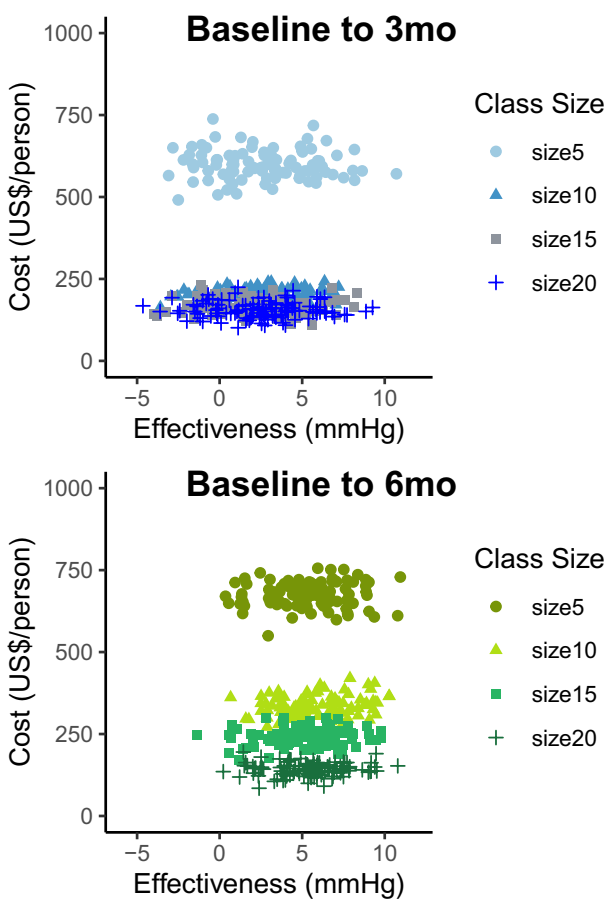
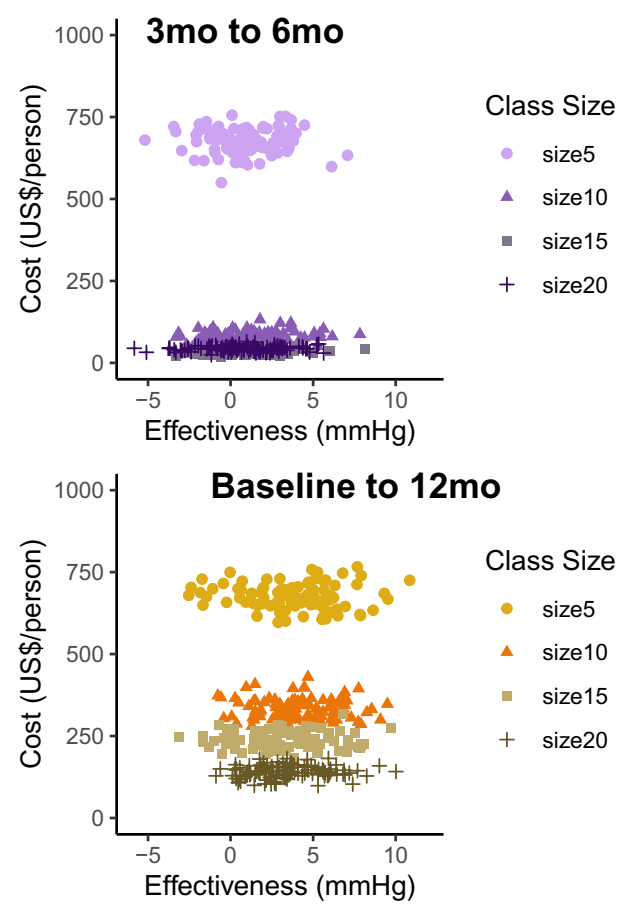

\section{Discussion}

Our analysis evaluated the costs and cost effectiveness of a 6-month culturally grounded physical activity intervention in Native Hawaiian adults with hypertension. To our knowledge, this is the first evaluation of the costs and cost effectiveness for a traditional cultural practice intended to improve blood pressure control among adults with hypertension. Our results extend existing evidence on the cost effectiveness of physical activity and educational interventions to control blood pressure by showing that cultural practices can be cost effective through within-trial retention 
and maintaining participation beyond the trial. Retaining $>80 \%$ of the participants contributed to the overall gains in clinical effectiveness by allowing participants to receive the full benefits of the intervention. The sustained participation beyond the 6-month intervention then helped keep blood pressure under control, on average, without additional costs. For a community-based organization in Hawai' ${ }^{i}$ with limited resources, offering Native Hawaiian cultural practices as a form of health promotion meets the needs of both their patients and their organizations when it is both clinically and cost effective, as well as culturally meaningful.

The reductions in systolic and diastolic blood pressure of $>3 \mathrm{mmHg}$ at 6 months aligns with other physical activity interventions that find between 0 and $10 \mathrm{mmHg}$ reductions over 6 months [30]. Similar to these interventions, hula reduced blood pressure for less than US $\$ 500 /$ person during the intervention timeframe [31], but is not cost saving. Medications and outpatient visits account for a significant portion of the costs related to controlling hypertension [32], but participants in the Ola Hou group continued medications throughout the 12-month follow-up [23]. Cost savings beyond 12 months would likely accrue with sustained blood pressure reduction that results in less medication use [13] or avoidance of secondary health outcomes, such as cardiovascular or cerebrovascular disease events [33]. A $5 \mathrm{mmHg}$ reduction in systolic blood pressure can lower a person's risk for ischemic heart disease by $21 \%$, stroke by $34 \%$, and all-cause mortality by $7 \%$ [28, 29]. Ola Hou has further been shown to improve health-related quality-of-life indices, such as bodily pain and social functioning [9], which may present additional opportunities for cost saving [34,35]. Measuring these outcomes through long-term data collection would likely contribute to the cost effectiveness of the program.

No established cost per outcome exists for hypertension treatment programs or physical activity interventions [14, 36]. For our 6-month intervention, a US $\$ 103 / \mathrm{mmHg}$ reduction in systolic blood pressure per hypertensive participant falls within existing estimates from educational and physical activity interventions of US $\$ 40-\mathrm{US} \$ 114 / \mathrm{mmHg}$ (systolic) $[18,33,37,38]$. The cost corresponds to a $67 \%$ probability that the intervention would be effective in reducing systolic blood pressure by $5 \mathrm{mmHg}$ at 6 months. Additional sensitivity analyses suggested that the intervention was insensitive to variation in the cost input parameters. Varying the costs from $20 \%$ below and above the expected intervention cost and increasing the number of participants helped to reduce the per-person costs only up to a point. Beyond 10 participants, the cost savings are marginal at 6 and 12 months and potentially not significantly different over time. The additional burden to recruit a larger class size through expanded outreach may offset the gains if recruitment costs exceed the per-person cost differences [39]. Increasing the class size also carries the risk of minimizing personalized support from instructors, which can compromise the achievement of health goals [40]. Personnel presented the largest cost; however, instructors provide essential, personalized feedback to reinforce lifestyle changes [41, 42]. Particularly for hula instruction, which requires an intimate knowledge of Hawaiian culture, tradition, and physical activity requirements, the cost of the Kumu Hula may misrepresent the effect of guided instruction on health outcomes.

Despite the established benefits of physical activity for hypertension-related health outcomes, at-risk adults tend not to maintain changes post-intervention [43, 44]. In part, time constraints $[45,46]$ and difficulty in achieving activity goals $[45,47]$ contribute to attenuation. A culturally grounded program may combat these barriers by combining social and cultural engagement with physical activity while providing opportunities to reinforce self-efficacy in achieving one's goals through shared experience [48, 49]. Engagement in familiar and routine practices require less social and cognitive strain than undertaking new activities [50]. Including self-regulation knowledge may further function to protect participants from reverting to pre-intervention habits that appear more likely in our control group. This is in addition to attending classes, which alone was not associated with a decrease in blood pressure [23]. While not cost effective, participants in the Ola Hou group maintained reduced blood pressure during the self-regulation component between months 3-6. At 12 months, we estimated that the Ola Hou group would maintain larger reductions in blood pressure compared with the control group, despite the reduced probability of being cost effective. Our findings indicate a need to further explore the relative influence of sociocultural engagement on blood pressure, including evaluating the change in quality of life over time [51].

The analyses benefited from using an intention-to-treat approach with individual-level data from an RCT. The intention-to-treat approach can reduce the potential to overestimate the effectiveness of an intervention compared with a complete-case analysis [52], while the individual data improved the precision of our effectiveness estimates by capturing group average reductions while minimizing the impact of potential influential observations. Our analysis was further strengthened by the use of objective blood pressure measurements taken through standard protocols to reduce issues associated with participant self-monitoring and response bias [53, 54].

Our analysis has several limitations. First, the relatively low enrollment of male participants limits the generalizability of our findings to men, but is not unique for lifestyle interventions with Native Hawaiians [55-57] or for group dance classes [58]. Next, we likely underestimated the cost of participation as participant costs were not included. Additionally, there was no evidence to support cost savings from medications or health service utilization throughout the 
intervention; however, these should be extrapolated from extant literature in future modeling studies to fully consider the provider's economic burden of hypertension [59]. Similarly, we were unable to directly examine the impact of the intervention on quality-of-life measures and refrained from estimating these given the time horizon of 12 months. Future studies should evaluate how including Native Hawaiianspecific quality-of-life estimates would affect the outcomes, including examining the lifetime cost effectiveness of secondary health outcomes related to hypertension, performing a cost-utility analysis with quality-adjusted life-years, and conducting a long-term implementation study of lifestyle programs. These analyses would better facilitate the comparison of the intervention across a wider range of interventions, such as those targeting medication adherence or diet.

\section{Conclusion}

Despite the additional cost compared with usual care, the Ola Hou intervention, which consists of an indigenous form of physical activity and self-regulation classes tailored to Native Hawaiian lifestyle practices, may be a cost-effective strategy for reducing blood pressure in adults with hypertension. Maintaining blood pressure reductions beyond the cessation of the intervention contributes to the potential cost effectiveness of the intervention. Community-based organizations and policy makers in Hawai ' 1 i should consider further evaluating the potential for indigenous forms of physical activity to reduce blood pressure, especially given the persistence of hypertension in Native Hawaiian people.

Supplementary Information The online version contains supplementary material available at https://doi.org/10.1007/s41669-021-00291-6.

Acknowledgements The authors would like to recognize and thank the community partners of the KāHOLO Project for insightful comments and interpretations. Specifically, Aukahi Seabury from I Ola Lāhui, Joey Gonsalves from Hui no Ke Ola Pono, Stacy Haumea from Mauli Ola, Geri Kaleponi, J. Kahaulahilahi Vegas from Wai' anae Coast Comprehensive Health Center, and Adrienne Dillard from Kula no na Po'e Hawai ${ }^{i} i$ for their thoughtful discussions. The authors are additionally grateful to Dedra Buchwald, Spero Manson, Jim Galvin, and discussions with others through the Native Alzheimer's Disease Resource Center for Minority Aging Research, as well as comments from the anonymous reviewers and the editor.

\section{Declarations}

Funding Financial support for this study was provided by a pilot award through the Native Alzheimer's Disease Resource Center for Minority Aging Research (NAD RCMAR), which is funded by the National Institute on Aging (Grant P30AG059295-01). The data for this study came from the KāHOLO Project, an RCT supported by the National Heart, Lung, and Blood Institute (Grant R01HL126577). The funding agreement ensured the authors' independence in designing the study, interpreting the data, and writing and publishing the report. The con- tents of this article are solely the responsibility of the authors and do not necessarily represent the official views of the National Heart, Lung, and Blood Institute and the National Institute on Aging of the National Institutes of Health.

Conflict of interest Ashley F. Railey, Clemma Muller, Carolyn Noonan, Maureen Schmitter-Edgecombe, Ka'imi Sinclair, Corin Kim, Mele Look, and J. Keawe'aimoku Kaholokula have declared no conflicts of interest.

Ethics approval This RCT was approved through the University of Hawai'i Institutional Review Board (IRB). The economic analysis used de-identified data.

Author contributions AFR, CM, ML, and JKK conceived the project and assisted with the protocol design. CK, JKK, and ML managed the trial, including recruitment and data collection. AFR performed statistical analyses and drafted the manuscript. ML, JKK, CK, CM, $\mathrm{CN}$, MSE, and KS interpreted the data, checked the statistical analyses, and edited the manuscript. All authors read, edited, and approved the final manuscript.

Patient consent Not required for publication.

Data availability statement Data and code are available upon reasonable request.

Open Access This article is licensed under a Creative Commons Attribution-NonCommercial 4.0 International License, which permits any non-commercial use, sharing, adaptation, distribution and reproduction in any medium or format, as long as you give appropriate credit to the original author(s) and the source, provide a link to the Creative Commons licence, and indicate if changes were made. The images or other third party material in this article are included in the article's Creative Commons licence, unless indicated otherwise in a credit line to the material. If material is not included in the article's Creative Commons licence and your intended use is not permitted by statutory regulation or exceeds the permitted use, you will need to obtain permission directly from the copyright holder. To view a copy of this licence, visit http://creativecommons.org/licenses/by-nc/4.0/.

\section{References}

1. Aluli NE, et al. Diabetes and cardiovascular risk factors in Native Hawaiians. Hawaii Med J. 2009;68:152-7.

2. Aluli NE, et al. All-cause and CVD mortality in Native Hawaiians. Diabetes Res Clin Pract. 2010;89:65-71.

3. Nakagawa K, Koenig MA, Seto TB, Asai SM, Chang CW. Racial disparities among Native Hawaiians and Pacific Islanders with intracerebral hemorrhage. Neurology. 2012;79:675-80.

4. Tung WC, Barnes M. Heart diseases among Native Hawaiians and Pacific Islanders. Home Health Care Manag Pract. 2014;26:110-3.

5. Johnson DB, Oyama N, LeMarchand L, Wilkens L. Native Hawaiians mortality, morbidity, and lifestyle: comparing data from 1982, 1990, and 2000. Pac Health Dialogues. 2004;11:120-30.

6. Schiller JS, Lucas JW, Peregoy JA. Summary health statistics for US adults: National Health Interview Survey, 2010. Vital Health Stat 2012; 10. Available at: https://www.cdc.gov/nchs/data/series/ sr_10/sr10_252.pdf.

7. Weintraub WS, et al. Value of primordial and primary prevention for cardiovascular disease: a policy statement from the American Heart Association. Circulation. 2011;124:967-90. 
8. Kaholokula JK, Ing CT, Look MA, Delafield R, Sinclair K. Culturally responsive approaches to health promotion for Native Hawaiians and Pacific Islanders. Ann Hum Biol. 2018;45:249-63.

9. Kaholokula JK, et al. Cultural dance program improves hypertension management for native Hawaiians and Pacific Islanders: a pilot randomized trial. J Racial Ethn Health Dispar. 2015;4:35-46.

10. Usagawa $\mathrm{T}$, et al. Metabolic equivalent determination in the cultural dance of hula. Int J Sports Med. 2014;35:399-402.

11. Kaholokula JK, et al. Kā-HOLO Project: a protocol for a randomized controlled trial of a native cultural dance program for cardiovascular disease prevention in Native Hawaiians. BMC Public Health. 2017;17:321.

12. Drummond ME, Sculpher MJ, Torrance G. Methods for the economic evaluation of health care programme. J Epidemiol Community Health. 2006;60(9):822-3. https://doi.org/10.1136/jech. 2005/040253corr2.

13. Richman IB, et al. Cost-effectiveness of intensive blood pressure management. JAMA Cardiol. 2016;1:872-9.

14. Zhang D, Wang G, Joo H. A systematic review of economic evidence on community hypertension interventions. Am J Prev Med. 2017;53:S121-30.

15. Murray CJL, et al. Effectiveness and costs of interventions to lower systolic blood pressure and cholesterol: a global and regional analysis on reduction of cardiovascular-disease risk. Lancet. 2003;361:717-25.

16. Kulchaitanaroaj $P$, et al. Cost-utility analysis of physician-pharmacist collaborative intervention for treating hypertension compared with usual care. J Hypertens. 2017;35:178-87.

17. Ramos M, et al. Cost-effectiveness of physical activity in the management of COPD patients in the UK. Int J COPD. 2019;14:227-39.

18. Neumann A, et al. The cost-effectiveness of interventions targeting lifestyle change for the prevention of diabetes in a Swedish primary care and community based prevention program. Eur J Health Econ. 2017;18:905-19.

19. Adashi EY, Geiger HJ, Fine MD. Health care reform and primary care-the growing importance of the community health center. $\mathrm{N}$ Engl J Med. 2010;362:2047-50.

20. Stillman AK, Tatar E, Kaeppler A. Hula Pahu: Hawaiian drum dances. Yearb Tradit Music. 1991. https://doi.org/10.2307/ 768403.

21. Stillman AK. Sacred hula: the historical hula a'la'apapa. Bishop Museum Press; 1998.

22. US Congress. United States Code: Native Hawaiian Health Care, 42 USC $\S \S 11701-11714$ (Suppl. 5 1988).

23. Kaholokula JK, et al. A cultural dance program improves hypertension control and cardiovascular disease risk: a randomized controlled trial. Ann Behav Med. 2021. https://doi.org/10.1093/ abm/kaaa127 (Epub 2 Mar 2021)

24. Pickering TG, et al. Recommendations for blood pressure measurement in humans and experimental animals: Part 1: blood pressure measurement in humans-a statement for professionals from the Subcommittee of Professional and Public Education of the American Heart Association. Circulation. 2005;111:697-716.

25. Husereau D, et al. Consolidated Health Economic Evaluation Reporting Standards (CHEERS) statement. BMJ. 2013;346:f1049.

26. Russell LB. Opportunity costs in modern medicine. Health Aff. 1992;11:162-9.

27. Briggs AH, Wonderling DE, Mooney CZ. Pulling cost-effectiveness analysis up by its bootstraps: a non-parametric approach to confidence interval estimation. Health Econ. 1997;6:327-40.

28. Appel LJ, et al. A clinical trial of the effects of dietary patterns on blood pressure. DASH Collaborative Research Group. N Engl J Med. 1997;336:1117-24.
29. Law M, Wald N, Morris J. Lowering blood pressure to prevent myocardial infarction and stroke: a new preventive strategy. Int J Technol Assess Health Care. 2005;21:145.

30. Noone C, et al. Comparative efficacy of exercise and anti-hypertensive pharmacological interventions in reducing blood pressure in people with hypertension: a network meta-analysis. Eur J Prev Cardiol. 2020;27:247-55.

31. Wu S, et al. Economic analysis of physical activity interventions. Am J Prev Med. 2011;40:149-58.

32. Dorans KS, Mills KT, Liu Y, He J. Trends in prevalence and control of hypertension according to the 2017 American College of Cardiology/American Heart Association (ACC/AHA) guideline. J Am Heart Assoc. 2018;7:e008888.

33. Hollenbeak CS, Weiner MG, Turner BJ. Cost-effectiveness of a peer and practice staff support intervention. Am J Manag Care. 2014;20:253-60.

34. Williamson JD, et al. Effect of intensive vs standard blood pressure control on probable dementia: a randomized clinical trial. JAMA. 2019;321:553-61.

35. Rehfeld K, et al. Dancing or fitness sport? The effects of two training programs on hippocampal plasticity and balance abilities in healthy seniors. Front Hum Neurosci. 2017;11:305.

36. Abu-Omar K, et al. The cost-effectiveness of physical activity interventions: a systematic review of reviews. Prev Med Rep. 2017;8:72-8.

37. Shireman T, Svarstad B. Cost-effectiveness of Wisconsin TEAM model for improving adherence and hypertension control in black patients. J Am Pharm Assoc. 2003;56:389-96.

38. Allen NB, et al. Blood pressure trajectories in early adulthood and subclinical atherosclerosis in middle age. JAMA. 2014;311:490-7.

39. Kakumanu $\mathrm{S}$, et al. Cost analysis and efficacy of recruitment strategies used in a large pragmatic community-based clinical trial targeting low-income seniors: a comparative descriptive analysis. Trials. 2019;20:577.

40. Rodrigues F, et al. Can interpersonal behavior influence the persistence and adherence to physical exercise practice in adults? A systematic review. Front Psychol. 2018;9:2141.

41. Philipsson A, Duberg A, Möller M, Hagberg L. Cost-utility analysis of a dance intervention for adolescent girls with internalizing problems. Cost Eff Resour Alloc. 2013;11:4.

42. Edmunds $\mathrm{K}$, et al. Cost-effectiveness analysis of supervised exercise training in men with prostate cancer previously treated with radiation therapy and androgen-deprivation therapy. Appl Health Econ Health Policy. 2020;10:11.

43. Sansano-Nadal O, et al. Exercise-based interventions to enhance long-term sustainability of physical activity in older adults: a systematic review and meta-analysis of randomized clinical trials. Int J Environ Res Public Health. 2019;16:2527.

44. Henderson RM, et al. Maintenance of physical function 1 year after exercise intervention in at-risk older adults: followup from the LIFE Study. J Gerontol Ser A Biol Sci Med Sci. 2018;73:688-94.

45. Gomersall SR, Norton K, Maher C, English C, Olds TS. In search of lost time: when people undertake a new exercise program, where does the time come from? A randomized controlled trial. J Sci Med Sport. 2015;18:43-8.

46. Brown H, Roberts J. Exercising choice: the economic determinants of physical activity behaviour of an employed population. Soc Sci Med. 2011;73(3):383-90. https://doi.org/10.1016/j.socsc imed.2011.06.001.

47. Gneezy U, Meier S, Rey-Biel P. When and why incentives (don't) work to modify behavior. J Econ Perspect. 2011;25:191-210.

48. Tabak RG, et al. A review of diabetes prevention program translations: use of cultural adaptation and implementation research. Transl Behav Med. 2015;5:401-14. 
49. Sinclair IA, et al. Outcomes from a diabetes self-management intervention for Native Hawaiians and Pacific People: partners in care. Ann Behav Med. 2013;45(1):24-32. https://doi.org/10.1007/ s12160-012-9422-1.

50. Kelly ME, et al. The impact ofsocial activities, social networks, social support and social relationships on the cognitive functioning of healthy older adults: a systematic review. Syst Rev. 2017;6(1):259.

51. Barrett S, Begg S, O'Halloran P, Kingsley M. Cost-effectiveness of telephone coaching for physically inactive ambulatory care hospital patients: economic evaluation alongside the Healthy4U randomised controlled trial. BMJ Open. 2019;9:1-8.

52. Hollis $\mathrm{S}$, Campbell $\mathrm{F}$. What is meant by intention to treat analysis? Survey of published randomised controlled trials. BMJ. 1999;319:670-4.

53. Prince SA, et al. A comparison of direct versus self-report measures for assessing physical activity in adults: a systematic review. Int J Behav Nutr Phys Act. 2008;5:56.

54. Stergiou GS, et al. Home blood pressure monitoring in the $21 \mathrm{st}$ century. J Clin Hypertens. 2018;20:1128-32.

55. Ing Townsend $\mathrm{C}$, et al. Comparing weight loss-maintenance outcomes of a worksite-based lifestyle program delivered via
DVD and face-to-face: a randomized trial. Health Educ Behav. 2018:45:569-80.

56. Keawe'aimoku Kaholokula J, Mau MK, Efird JT, Leake A, West M, Palakiko DM, et al. A family and community focused lifestyle program prevents weight regain in pacific islanders: a pilot randomized controlled trial. Health Edu Behav. 2012;39:386-95.

57. Townsend CKM, et al. The PILI@Work Program: a translation of the diabetes prevention program to Native Hawaiian-serving worksites in Hawai 'i. Transl Behav Med. 2016;6:190-201.

58. Merom D, et al. Cognitive benefits of social dancing and walking in old age: the dancing mind randomized controlled trial. Front Aging Neurosci. 2016;8:26.

59. Huang SJ, Galárraga O, Smith KA, Fuimaono S, McGarvey ST. Cost-effectiveness analysis of a cluster-randomized, culturally tailored, community health worker home-visiting diabetes intervention versus standard care in American Samoa. Hum Resour Health. 2019;17:1-9.

60. Hawai 'i Commercial Real Estate, L. Honolulu office market reports. 2015. Available at: https://hawaiicre.com/market-repor ts/. Accessed 15 June 2020. 